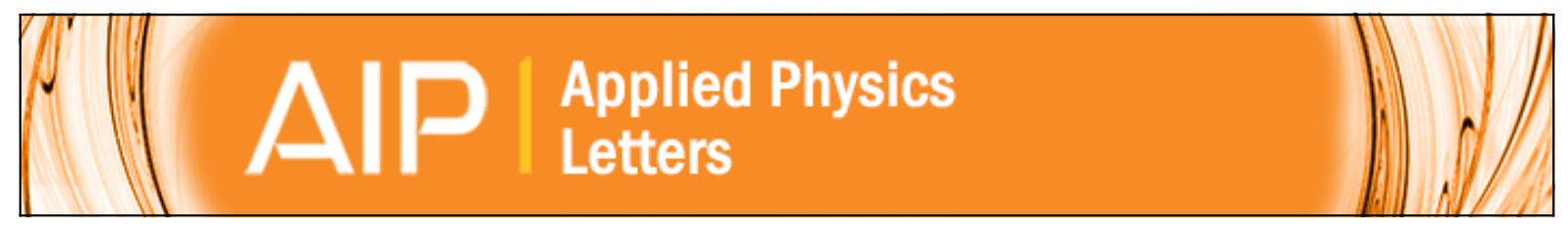

\title{
Photoluminescent characteristics of Ni-catalyzed GaN nanowires
}

Jinkyoung Yoo, Young-Joon Hong, Sung Jin An, Gyu-Chul Yi, Bonghwan Chon, Taiha Joo, Jong-Wook Kim, and Jeong-Soo Lee

Citation: Applied Physics Letters 89, 043124 (2006); doi: 10.1063/1.2243710

View online: http://dx.doi.org/10.1063/1.2243710

View Table of Contents: http://scitation.aip.org/content/aip/journal/apl/89/4?ver=pdfcov

Published by the AIP Publishing

\section{Articles you may be interested in}

Optical properties of functionalized GaN nanowires

J. Appl. Phys. 109, 053523 (2011); 10.1063/1.3552919

Injection-level-dependent internal quantum efficiency and lasing in low-defect GaN nanowires

J. Appl. Phys. 109, 044312 (2011); 10.1063/1.3553418

Optical and structural study of GaN nanowires grown by catalyst-free molecular beam epitaxy. II. Sub-band-gap luminescence and electron irradiation effects

J. Appl. Phys. 101, 113506 (2007); 10.1063/1.2736266

Optical and structural study of GaN nanowires grown by catalyst-free molecular beam epitaxy. I. Near-band-edge luminescence and strain effects

J. Appl. Phys. 101, 113505 (2007); 10.1063/1.2736264

Quantum confinement of excitons in dendrite-like GaN nanowires

J. Appl. Phys. 98, 086104 (2005); 10.1063/1.2084334

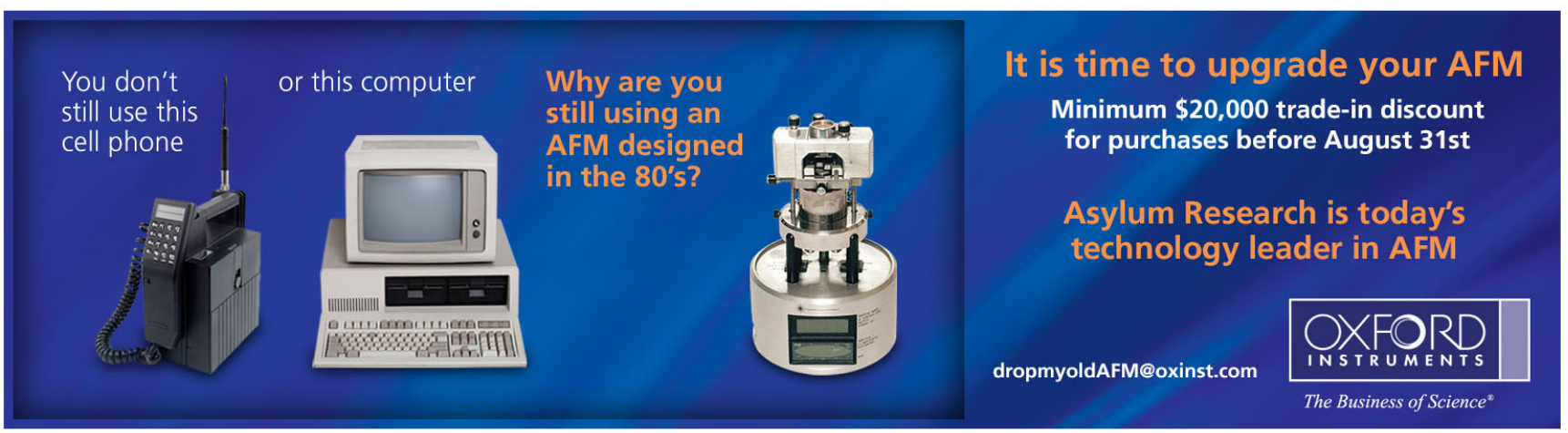




\title{
Photoluminescent characteristics of Ni-catalyzed GaN nanowires
}

\author{
Jinkyoung Yoo, Young-Joon Hong, Sung Jin An, and Gyu-Chul $\mathrm{Yj}^{\mathrm{a}}{ }^{\mathrm{j}}$ \\ National CRI Center for Semiconductor Nanorods, Pohang University of Science and Technology \\ (POSTECH), San-31 Hyoja-dong, Pohang, Gyeongbuk 790-784, Korea and Department of Materials Science \\ and Engineering, Pohang University of Science and Technology (POSTECH), San-31 Hyoja-dong, \\ Pohang, Gyeongbuk 790-784, Korea
}

\begin{abstract}
Bonghwan Chon and Taiha Joo
Division of Molecular and Life Sciences, Pohang University of Science and Technology (POSTECH), San-31 Hyoja-dong, Pohang, Kyungbuk 790-784, Korea and Department of Chemistry, Pohang University of Science and Technology (POSTECH), San-31 Hyoja-dong, Pohang, Kyungbuk 790-784, Korea
\end{abstract}

Jong-Wook Kim and Jeong-Soo Lee

LED R\&D Laboratory, LG Electronics Institute of Technology, 16 Woomyeon-Dong, Seocho-Gu, Seoul 137-724, Korea

(Received 12 April 2006; accepted 6 July 2006; published online 28 July 2006)

\begin{abstract}
The authors report on time-integrated and time-resolved photoluminescence (PL) of GaN nanowires grown by the Ni-catalyst-assisted vapor-liquid-solid method. From PL spectra of Ni-catalyzed GaN nanowires at $10 \mathrm{~K}$, several PL peaks were observed at 3.472, 3.437, and $3.266 \mathrm{eV}$, respectively. PL peaks at 3.472 and $3.266 \mathrm{eV}$ are attributed to neutral-donor-bound excitons and donor-acceptor pair, respectively. Furthermore, according to the results from temperature-dependent and time-resolved PL measurements, the origin of the PL peak at $3.437 \mathrm{eV}$ is also discussed. (C) 2006 American Institute of Physics. [DOI: 10.1063/1.2243710]
\end{abstract}

One-dimensional GaN nanostructures including nanowires, nanorods, and nanotubes have recently attracted much attention because of their potential applications for optoelectronic devices in the nanoscale. ${ }^{1,2} \mathrm{GaN}$ nanowires have been synthesized by many different nanowire growth methods. ${ }^{3-6}$ Among numerous nanowire synthesis methods, the metal catalyst-assisted vapor-liquid-solid (VLS) growth method has been widely employed because this technique offers easy and size-controllable growth of many semiconductor nanowires. However, since the metal catalysts used for nanowire growth may act as impurities in the nanomaterial and even a small amount of impurities in semiconductors can significantly change physical properties of the host materials, it is very important to characterize the impurities and defects in catalyst-assisted grown semiconductor nanomaterials. The effect of impurities and defects on the physical properties of host materials is also expected to increase with a reduction in the size of the materials. In addition, the unintentionally doped impurities in semiconductors make it difficult to accurately control their conductivities and optical properties. Nevertheless, the defect characterization of catalyst-assisted grown semiconductor nanowires has rarely been reported because of difficulties in elemental analysis and electrical device fabrications. ${ }^{7}$ Meanwhile, optical characterization methods such as photoluminescence (PL) spectroscopy requiring no physical contacts are useful for defect characterization of the nanomaterials. ${ }^{8}$ In particular, low temperature PL spectroscopy is a very sensitive and nondestructive tool for characterizing radiative defects in semiconductors. ${ }^{9}$ Although a few papers on synthesis and PL spectra of GaN nanowires have previously been reported, ${ }^{10}$ near-band-edge (NBE) PL peak positions from catalyst-assisted grown $\mathrm{GaN}$ nanowires have not been consistent with those of epitaxial thin films. ${ }^{5,6}$ Furthermore, time-resolved PL (TRPL) spectroscopy enables ${ }^{a)}$ Author to whom correspondence should be addressed; electronic mail:
gcyi@ postech.ac.kr the investigation of exciton lifetime, an important parameter related to defects in materials and device performance. Despite the importance of TRPL measurements, TRPL behavior in $\mathrm{GaN}$ nanowires has rarely been reported. ${ }^{11}$ In this letter, we report on both time-integrated and time-resolved photoluminescent properties of $\mathrm{GaN}$ nanowires grown by the $\mathrm{Ni}$ catalyst-assisted VLS method.

$\mathrm{GaN}$ nanowires were grown on a 2 -nm-thick Ni layer coated $\mathrm{Al}_{2} \mathrm{O}_{3}(1-102)$ substrates using a low pressure metalorganic vapor phase epitaxy (MOVPE) system. For GaN nanowire growth, trimethyl-gallium (TMGa) and ammonia $\left(\mathrm{NH}_{3}\right)$ were employed as reactants and hydrogen was used as a carrier gas. Prior to GaN nanowire growth, a 2-nm-thick Ni layer was deposited on the $r$-sapphire substrates using a high vacuum e-beam evaporator, and susbsequently annealed at $800{ }^{\circ} \mathrm{C}$ for 10 min under 100 Torr in order to form Ni droplets which were used as catalytic sites for nanowire growth. During GaN nanowire growth, typical TMGa and $\mathrm{NH}_{3}$ flow rates were 3.5 and 300 SCCM (SCCM denotes cubic centimeter per minute at STP), respectively. The growth temperature was in the range of $750-850{ }^{\circ} \mathrm{C}$.

Both time-integrated and time-resolved PL spectra of the GaN nanowires were measured in order to investigate optical properties of the nanowires and radiative recombination related to defects in the nanomaterials. The time-integrated PL (TIPL) measurements were performed at various temperatures in the range of $10-300 \mathrm{~K}$ with the $325 \mathrm{~nm}$ line of a continuous wave $\mathrm{He}-\mathrm{Cd}$ laser used as an excitation source. Details of the TIPL measurements have previously been reported elsewhere. ${ }^{9,12}$ Meanwhile, a TRPL measurement system consists of a femtosecond Ti:sapphire oscillator, a frequency tripler, and a time correlated single photon counting system employing a microchannel plate photomultiplier tube. The excitation source for TRPL measurements was a femtosecond Ti:sapphire laser laser pumped by a diode pumped neodymium-doped yttrium orthovaradate vanadate laser. The Ti:sapphire laser can provide $100 \mathrm{fs}$ pulses at a repetition 

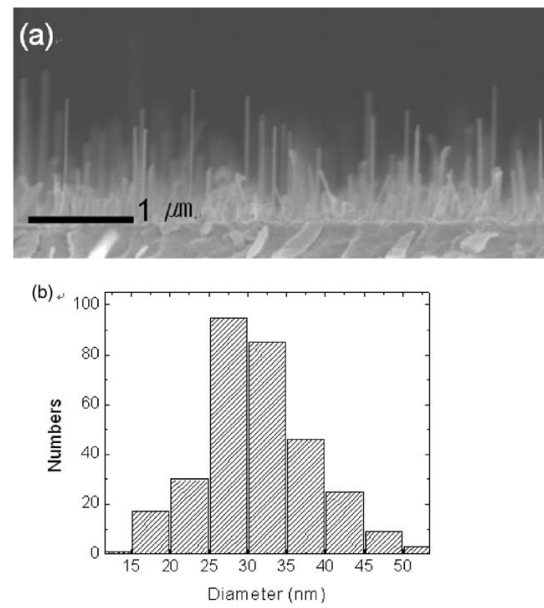

FIG. 1. (a) Scanning electron microscopic image of GaN nanowires grown on $r$-plane sapphire substrates and (b) histogram of GaN nanowire diameters. The electron microscopic image shows that $\mathrm{GaN}$ nanowires with a typical mean diameter $(30 \pm 10 \mathrm{~nm})$ of $\mathrm{GaN}$ nanowires are vertically aligned on the $r$-sapphire substrate.

rate of $82 \mathrm{MHz}$. Output of the femtosecond Ti:sapphire laser (Tsunami, Spectra-Physics) at $800 \mathrm{~nm}$ was tripled to $266 \mathrm{~nm}$ to serve as an excitation source for the TRPL experiments. The PL signal was confocally collected and TRPL was measured by a time correlated single photon counting system, which provides sub-10 ps time resolution with deconvolution. The resolution of TRPL spectra was $0.1 \mathrm{meV}$. All TRPL measurements in this study were carried out at $10 \mathrm{~K}$.

Field emission scanning electron microscopy revealed the general morphology of GaN nanowires. Figure 1(a) clearly shows that $\mathrm{GaN}$ nanowires were vertically grown on $r$-plane sapphire substrates. The mean diameter of $\mathrm{GaN}$ nanowires was $30 \pm 10 \mathrm{~nm}$ and nanowire length showed wide distribution in the range of $800 \pm 300 \mathrm{~nm}$, as shown in Fig. 1(b). A typical nanowire density was $1.5 \times 10^{9} / \mathrm{cm}^{2}$.

Defect-related NBE emissions from the GaN nanowires were investigated using low temperature PL spectroscopy. As shown in Fig. 2, a typical $10 \mathrm{~K}$ PL spectrum of Ni-catalyzed $\mathrm{GaN}$ nanowires exhibited a strong ultraviolet emission while the deep level emission at $2.2-2.5 \mathrm{eV}$, related to structural defects or impurities, was too weak to be observed. Although previous reports on PL spectra of GaN nanowires grown by catalyst-assisted methods did not show well-resolved PL peaks in NBE emission spectra, ${ }^{3-6,10}$ the NBE emission in

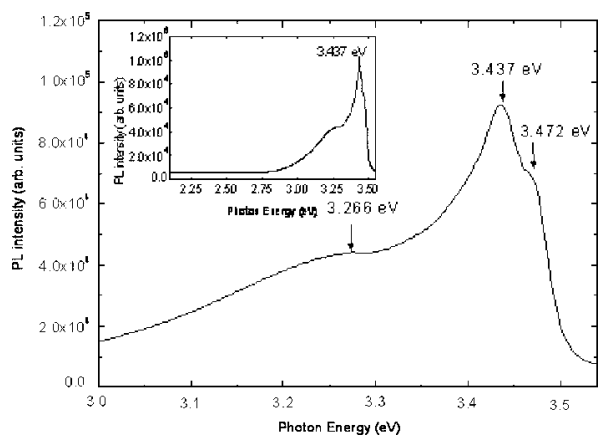

FIG. 2. TIPL spectra of Ni-catalyzed GaN nanowires at $10 \mathrm{~K}$. The NBE emission was clearly resolved into three PL peaks at $3.472,3.437$, and $3.266 \mathrm{eV}$, respectively. Each dominant PL peak at 3.472 and $3.266 \mathrm{eV}$ is tentatively attributed to the neutral-donor-bound excitons $\left(I_{2}\right)$ and the donoracceptor nair (DAP) recombination respectively. The inset shows that the deep level emission is too weak to be observed.
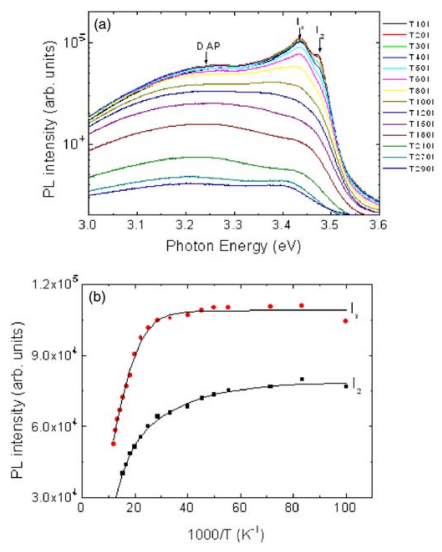

FIG. 3. (Color online) (a) Temperature-dependent TIPL spectra at various temperatures in the range of $10 \mathrm{~K}$ and room temperature and (b) the Arrehnius plot of the emission intensities at 3.472 and $3.437 \mathrm{eV}$ under various temperatures. The emission intensities of NBE emission peaks decreased with increasing temperature, showing thermal quenching effect. From the relationship between emission integrated intensity and temperature, the ionization energies of the bound excitons were estimated to be 5.1 and $17.1 \mathrm{meV}$ for the PL peaks at 3.472 and $3.437 \mathrm{eV}$, respectively.

Fig. 2 was clearly resolved into three PL peaks at 3.472 , 3.437 , and $3.266 \mathrm{eV}$. The PL peaks at 3.472 and $3.266 \mathrm{eV}$ have previously been observed for many bulk $\mathrm{GaN}$ materials, attributed to neutral-donor-bound exciton $\left(I_{2}\right)$ and donoracceptor-pair (DAP) transition, respectively. ${ }^{13-15}$

It is also notable that the dominant emission of our $\mathrm{GaN}$ nanowires grown by Ni-catalyst-assisted MOVPE was observed at $3.437 \mathrm{eV}$ with additional peaks at 3.472 and $3.266 \mathrm{eV}$ although the dominant emission peak of $\mathrm{GaN}$ nanowires previously grown by other groups has previously been observed at $3.2-3.35 \mathrm{eV}^{3-6,8}$ As mentioned above, the PL peaks at 3.472 and $3.266 \mathrm{eV}$ are commonly observed from many epitaxial films grown by MOVPE, associated with defects presumably due to the same growth process. Meanwhile, the new PL peak at $3.437 \mathrm{eV}$ is not well known for epitaxial GaN films, so it may result from Ni-related defects. No report has been made about the PL peak at $3.437 \mathrm{eV}$ for high quality GaN bulk materials. Only a PL peak at $3.41 \mathrm{eV}$ has been observed and tentatively attributed to strongly, localized excitons in structural defects, such as stacking faults or screw dislocations, ${ }^{16}$ and a PL peak at $3.44 \mathrm{eV}$ to neutral acceptor-bound excitons. ${ }^{17,18}$ Since the GaN nanowires prepared in this study have not shown dislocations or stacking faults, similar to the previous report, ${ }^{6,10}$ the PL peak at $3.437 \mathrm{eV}$ is expected to result from strongly localized excitons, presumably neutral acceptor-bound excitons.

The PL peaks tentatively assigned to excitonic emissions were investigated by temperature-dependent PL and timeresolved PL measurements. As shown in Fig. 3(a), $I_{2}$ and $I_{x}$ peak intensities decrease with increasing temperature and the peaks disappeared around 50 and $100 \mathrm{~K}$, respectively. This thermal quenching can be explained in terms of the decomposition of bound excitons to free excitons. However, the thermal quenching rates of $I_{2}, I_{x}$, and DAP emission were different from each other because of different exciton ionization energies. The exciton ionization energies of $I_{2}$ and $I_{x}$ of $\mathrm{Ni}$-catalyzed $\mathrm{GaN}$ nanowires were determined by the Arrhenius plot of integrated PL intensities of the $I_{2}$ and $I_{r}$, that is, the following equation: ${ }^{13}$ 


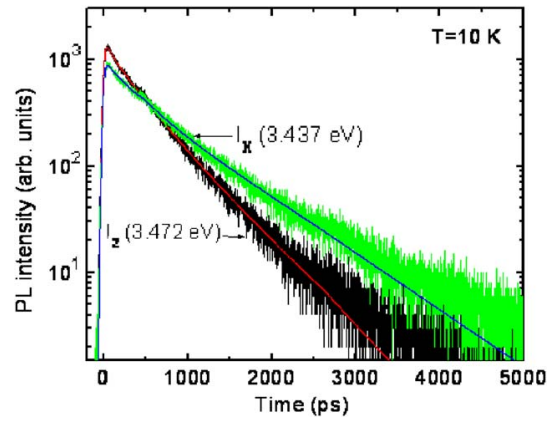

FIG. 4. (Color online) TRPL spectra of Ni-catalyzed GaN nanowires at $10 \mathrm{~K}$. Each decay profile was measured at 3.472 and $3.437 \mathrm{eV}$, respectively. All decay profiles fitted well with a double exponential decay curve.

$$
I=\frac{I_{o}}{1+A \exp \left(-E_{\mathrm{fx}} / k_{B} T\right)+B \exp \left(-E_{\mathrm{bx}} / k_{B} T\right)},
$$

where $E_{\mathrm{fx}}$ and $E_{\mathrm{bx}}$ are the ionization energy of the free exciton and the bound exciton in GaN, respectively, and $A$ and $B$ are coefficients. In Fig. 3(b), the solid lines are the leastsquare-fits to their variations. From the fits, the $E_{\mathrm{fx}}$ and the $E_{\mathrm{bx}}$ for $3.472 \mathrm{eV}$ peak are determined to be 28.3 and $5.1 \mathrm{meV}$, respectively. For $I_{2}, E_{\mathrm{bx}}$ of $\mathrm{GaN}$ nanowires is slightly smaller than that of undoped GaN epitaxial films, $6.2 \pm 1.1 \mathrm{meV} .^{18}$ Meanwhile, from the $I_{x}$ fit, the $E_{\mathrm{fx}}$ and the $E_{\mathrm{bx}}$ for $3.437 \mathrm{eV}$ peak are estimated to be 27.2 and $17.1 \mathrm{meV}$, respectively. The $E_{\mathrm{bx}}$ for $I_{x}$ is larger than that (11 meV) of exciton bound to $\mathrm{Mg}$ and smaller than that $(22 \mathrm{meV})$ of exciton bound to $\mathrm{Zn} .^{19}$

TRPL of Ni-catalyzed GaN nanowires were measured to investigate dynamics of bound excitons in $\mathrm{GaN}$ nanowires. Figure 4 shows the TRPL data obtained at 3.469 and $3.437 \mathrm{eV}$, which correspond to $I_{2}$ and $I_{x}$, respectively. All observed TRPL data were fitted well with a double exponential decay curve. The double exponential decay suggests that two different decay and capture processes are involved in the emission. The decay time constants of $I_{2}$ are estimated to be 197 and 526 ps. For $I_{x}$, however, the decay time constants, 252 and $816 \mathrm{ps}$, were larger than those of $I_{2}$. The decay time constants of $I_{x}$ are similar to those of neutral acceptor-bound exciton in homoepitaxial thin film, ${ }^{20} 800 \mathrm{ps}$, and $450 \mathrm{ps}$ for Mg-doped GaN. ${ }^{21}$

According to the TIPL and TRPL measurements of GaN nanowires grown by the Ni-catalyst-assisted VLS method, the origin of $I_{x}$ emissions should be related to the bound excitons because the $I_{x}$ emission peak position and the ionization energy of the bound excitons are similar to those of acceptor-bound excitons in GaN bulk materials. This result implies that a large amount of acceptors exist in GaN nanowires. The possible acceptors in Ni-catalyzed GaN nanowires are $\mathrm{Ni}$, Ga vacancy $\left(V_{\mathrm{Ga}}\right)$, and $\mathrm{C}$. Among these possible acceptors, $\mathrm{Ni}$ in the form of $\mathrm{Ni}_{\mathrm{Ga}}^{2+}$ is the most probable acceptor in the $\mathrm{GaN}$ nanowires because $\mathrm{Ni}$ metal particles were used for the nanowire growth in the MOVPE reactor. So, Ni can exist in the material up to a Ni solubility limit. This is consistent with the previous report that the valence state of $\mathrm{Ni}$ in Ni-implanted $\mathrm{GaN}$ thin film is $+2 .^{22}$ Furthermore, several results on transition metal implanted GaN thin film have reported that $\mathrm{Ni}$ incorporation enhances hole concentration. $^{23}$ Accordingly, $\mathrm{Ni}$ may be incorporated into the GaN nanowires and generate an acceptor state. From this point of view, the quantitative analysis of impurities in semiconductor nanowires is very important. Nevertheless, the incorporation of metal catalysts in nanowires during the VLS process has rarely been investigated. Although TEM is a very powerful technique, the limit of TEM elemental analysis is not as good as approximately $1 \%$. Here, we demonstrate that both TIPL and TRPL measurements can be used to characterize the defects in semiconductor nanomaterials.

In summary, we performed both TIPL and TRPL measurements of $\mathrm{GaN}$ nanowires grown by a Ni-catalyst-assisted VLS method. Three near-band-edge emission peaks from TIPL spectra of the GaN nanowires were observed at 3.472, 3.437 , and $3.266 \mathrm{eV}$, attributed to the neutral-donor-bound exciton, neutral-acceptor-bound exciton, and donor-acceptor pair peaks. From the temperature-dependent and timeresolved PL measurements, the origin of the acceptors in the $\mathrm{GaN}$ nanowires was considered to result from $\mathrm{Ni}$ catalyst metals.

This work was financially supported through the National Creative Research Initiative Project by the Korea Science and Engineering Foundations (KOSEF) and LG Electronics Institute of Technology.

${ }^{1}$ H.-M. Kim, T. W. Kang, and K. S. Chung, Adv. Mater. (Weinheim, Ger.) 15, 567 (2003).

${ }^{2}$ F. Qian, S. Gradečak, Y. Li, C. Wen, and C. M. Lieber, Nano Lett. 5, 2287 (2005).

${ }^{3}$ H. Weiqiang, F. Shoushan, L. Qunqing, and H. Yongdan, Science 277, 1287 (1997).

${ }^{4}$ H.-M. Kim, D. S. Kim, Y. S. Park, D. Y. Kim, T. W. Kang, and K. S. Chung, Adv. Mater. (Weinheim, Ger.) 14, 991 (2002).

${ }^{5}$ T. Kuykendall, P. J. Pauzauskie, Y. Zhang, J. Goldberger, D. Sirbuly, J. Denlinger, and P. Yang, Nat. Mater. 3, 524 (2004).

${ }^{6}$ G. Seryogin, I. Shalish, W. Moberlychan, and V. Narayanamurti, Nanotechnology 16, 2342 (2005).

${ }^{7}$ D. E. Perea, J. E. Allen, S. J. May, B. W. Wessels, D. N. Seidman, and L. J. Lauhon, Nano Lett. 6, 181 (2006).

${ }^{8}$ W. I. Park, Y. H. Jun, S. W. Jung, and G.-C. Yi, Appl. Phys. Lett. 82, 964 (2003).

${ }^{9}$ G. D. Gilliland, Mater. Sci. Eng., R. 18, 99 (1997).

${ }^{10}$ B. Ha, S. H. Seo, J. H. Cho, C. S. Yoon, J. Yoo, G.-C. Yi, C. Y. Park, and C. J. Lee, J. Phys. Chem. B 109, 11095 (2005).

${ }^{11}$ Y. S. Park, J. H. Na, R. A. Taylor, C. M. Park, K. H. Lee, and T. W. Kang, Nanotechnology 17, 913 (2006).

${ }^{12}$ S. Hong, T. Joo, W. I. Park, Y. H. Jun, and G.-C. Yi, Appl. Phys. Lett. 83, 4157 (2003).

${ }^{13}$ M. Leroux, N. Grandjean, B. Beaumont, G. Nataf, F. Semond, J. Massies, and P. Gibart, J. Appl. Phys. 86, 3721 (1999).

${ }^{14}$ D. G. Chtchekine, Z. C. Feng, S. J. Chua, and G. D. Gilliland, Phys. Rev. B 63, 125211 (2001).

${ }^{15}$ S. Strauf, S. M. Ulrich, P. Michler, J. Gutowski, T. Böttcher, S. Figge, S. Einfeldt, and D. Hommel, Phys. Status Solidi B 228, 379 (2001).

${ }^{16}$ M. Albrecht, S. Christiansen, G. Salviati, C. Zanotti-Fregonara, Y. T. Rebane, Y. G. Shreter, M. Mayer, A. Pelzmann, M. Kamp, K. J. Ebeling, M. D. Bremser, R. F. Davis, and H. P. Strunk, Mater. Res. Soc. Symp. Proc. 468, 293 (1997).

${ }^{17}$ B. Monemar, J. Phys.: Condens. Matter 13, 7011 (2001).

${ }^{18}$ D. Volm, K. Oettinger, T. Streibl, D. Kovalev, M. Ben-Chorin, J. Diener, B. K. Meyer, J. Majewski, L. Eckey, A. Hoffmann, H. Amano, I. Akasaki, K. Hiramatsu, and T. Detchprohm, Phys. Rev. B 53, 16543 (1996).

${ }^{19}$ K. P. Korona, R. Doradziński, M. Palczewska, M. Pietras, M. Kamińska, and J. Kuhl, Phys. Status Solidi B 235, 40 (2003).

${ }^{20}$ K. P. Korona, Phys. Rev. B 65, 235312 (2002).

${ }^{21}$ M. Smith, G. D. Chen, J. Y. Lin, H. X. Jiang, M. Asif Khan, and C. J. Sun, Appl. Phys. Lett. 67, 3295 (1995).

${ }^{22}$ R.-T. Huang, C.-F. Hsu, J.-J. Kai, F.-R. Chen, and T.-S. Chin, Appl. Phys. Lett. 87, 202507 (2005).

${ }^{23}$ I. Waki, H. Fujioka, M. Oshima, H. Miki, and A. Fukizawa, Appl. Phys. Lett. 78, 2899 (2001). 